\title{
Comparison of Non-Invasive Clinical Algorithms for Liver Fibrosis in Patients With Chronic Hepatitis B to Reduce the Need for Liver Biopsy: Application of Enhanced Liver Fibrosis and Mac-2 Binding Protein Glycosylation Isomer
}

\author{
Mina Hur (i), M.D., Ph.D. ${ }^{1}$, Mikyoung Park 타, M.D., Ph.D. ${ }^{2}$, Hee-Won Moon 타, M.D., Ph.D. ${ }^{1}$, \\ Won Hyeok Choe (10), M.D., Ph.D. ${ }^{3}$, and Chae Hoon Lee 타, M.D., Ph.D. ${ }^{2}$ \\ ${ }^{1}$ Department of Laboratory Medicine, Konkuk University School of Medicine, Seoul, Korea; ${ }^{2}$ Department of Laboratory Medicine, Yeungnam University \\ College of Medicine, Daegu, Korea; ${ }^{3}$ Department of Internal Medicine, Konkuk University School of Medicine, Seoul, Korea
}

Background: Non-invasive clinical algorithms for the detection of liver fibrosis (LF) can reduce the need for liver biopsy (LB). We explored the implementation of two serum biomarkers, enhanced liver fibrosis (ELF) and Mac-2 binding protein glycosylation isomer (M2BPGi), in clinical algorithms for LF in chronic hepatitis B (CHB) patients.

Methods: Two clinical algorithms were applied to 152 CHB patients: (1) transient elastography (TE) followed by biomarkers (TE/ELF and TE/M2GPGi); (2) biomarker test followed by TE (ELF/TE and M2BPGi/TE). Using the cut-off value or index for the detection of advanced LF (TE $\geq F 3$; 9.8 in ELF and 3.0 in M2BPGi), LB was expected to be performed in cases with discordant $\mathrm{TE}$ and biomarker results.

Results: In both algorithms, the expected number of LBs was lower when using M2BPGi than when using ELF (TE/ELF or ELF/TE, 13.2\% [N=20]; TE/M2BPGi or M2BPGi/TE, 9.9\% $[N=15])$, although there was no statistical difference $(P=0.398)$. In the TE low-risk group $(\mathrm{TE} \leq \mathrm{F} 2)$, the discordance rate was significantly lower in the TE/M2BPGi approach than in the TE/ELF approach (1.5\% [2/136] vs. $11.0 \%$ [15/136], $P=0.002)$. In the biomarker low-risk group, there was no significant difference between the ELF/TE and M2BPGi/TE approaches (3.9\% [5/126] vs. 8.8\% [13/147], $P=0.118$ ).

Conclusions: Both ELF and M2BPGi can be implemented in non-invasive clinical algorithms for assessing LF in CHB patients. Given the lowest possibility of losing advanced LF cases in the low-risk group when using the TE/M2BPGi approach, this combination seems useful in clinical practice.

Key Words: Enhanced liver fibrosis, Mac-2 binding protein glycosylation isomer, Clinical algorithm, Liver fibrosis, Chronic hepatitis B
Received: June 2, 2021

Revision received: August 20, 2021

Accepted: September 10, 2021

\section{Corresponding author:}

Mikyoung Park, M.D., Ph.D.

Department of Laboratory Medicine, Yeungnam University College of Medicine, Yeungnam University Medical Center, 170 Hyunchoong-ro, Nam-gu,

Daegu 42415, Korea

Tel: +82-53-640-6703

Fax: +82-53-627-1093

E-mail: mikyoung.pak@gmail.com

\section{INTRODUCTION}

Various diseases in different organs are associated with fibrosis through a common pathogenic pathway [1]. Acute and chronic inflammation can lead to fibrosis by inducing epithelial cell injury resulting in fibrogenic effector cell activation and prolifera- 
tion [1]. Chronic hepatitis B (CHB), affecting approximately 350 million people worldwide, is among the most common causes of chronic liver injury [2, 3]. Chronic inflammation due to CHB affects hepatocytes or biliary cells, activates fibrogenic effector cells, and leads to the deposition of extracellular matrix (ECM). In response to injury and excess ECM synthesis, hepatic stellate cells are transformed into myofibroblasts [1]. Liver fibrosis (LF) can cause liver cirrhosis (LC), leading to portal hypertension, variceal hemorrhage, and ascites, and even hepatocellular carcinoma in some cases. As hepatocytes are capable of regeneration, LF is a dynamic process that can be reversed by therapeutic intervention $[1,3]$.

Liver biopsy (LB), the reference method for diagnosing LF, has several limitations, including invasiveness, high cost, sampling error, and inter-observer variability in pathologic interpretation [4-7]. Therefore, non-invasive approaches have been developed to assess LF [4, 7]. Transient elastography (TE) using the FibroScan (Echosens, Paris, France) is widely used for grading LF in routine clinical practice, but TE results can be affected by the technique, obesity, ascites, or liver mass [8-11].

Recently, novel biomarkers for assessing LF have been introduced into clinical practice. The enhanced liver fibrosis (ELF) score is a set of circulating markers of hepatic matrix metabolism comprising hyaluronic acid (HA), tissue inhibitor of matrix metalloproteinases-1 (TIMP-1), and aminoterminal propeptide of procollagen type III (PIIINP) [4, 7, 12]. Mac-2 binding protein glycosylation isomer (M2BPGi) is a glycoprotein secreted by hepatic stellate cells during LF progression [13-17]. ELF and M2BPGi have comparable performance for assessing LF and are significantly associated with fibrosis stages 2 and 3 in $\mathrm{CHB}$ patients [18, 19]. Non-invasive clinical algorithms using TE in combination with serum biomarkers for assessing LF in various liver diseases have been suggested [20-24]. Tapper and Lok [25] suggested a sequential approach using TE and a serum biomarker to detect advanced liver diseases; in cases of indeterminate or discordant TE and serum biomarker results, LB should be performed in advanced liver diseases. Yoneda, et al. [26] suggested that intermediate and high risk of LF based on serum biomarkers and moderate and severe LF based on TE indicate a need to perform LB in non-alcoholic fatty liver diseases (NAFLDs). The 2021 NAFLD clinical practice guidelines from the Korean Association for the Study of the Liver suggest a non-invasive clinical algorithm that considers additional serological test, such as ELF and M2BPGi, radiologic test, or LB, following the evaluation of TE, fibrosis-4 (FIB-4), or NAFLD fibrosis score (NFS) [27].
To the best of our knowledge, no study has compared non-invasive clinical algorithms to assess LF using ELF and M2BPGi. We implemented these two novel biomarkers in newly proposed non-invasive clinical algorithms for LF in CHB patients [25]. We hypothesized that sequential approaches combining a serum biomarker and TE may significantly reduce the number of LBS. We explored how much these clinical algorithms would reduce the number of LBs and how high the discordance rate between biomarkers and TE would be, especially in the low-risk group, for each sequential approach.

\section{MATERIAS AND METHODS}

\section{Study population}

The study population consisted of $152 \mathrm{CHB}$ patients who were admitted to Konkuk University Medical Center (KUMC), Seoul, Korea between October 2016 and February 2017. For 123 of these patients, data were derived from a previous study [18], and 29 patients were additionally enrolled. The patients visited the liver clinic and completed TE and blood sampling as a routine clinical practice. The patients were diagnosed as having $\mathrm{CHB}$ prior to study enrollment and received antiviral treatment. Patients with human immunodeficiency virus infection and other hepatic diseases, such as hepatitis A, hepatitis C, autoimmune hepatitis, alcoholic liver diseases, or NAFLDs, were excluded (Supplemental Data Fig. 1).

LB was not performed, but simulated using the clinical algorithms, and no additional intervention or blood sampling was performed; therefore, this retrospective study with forward sample collection was exempted from approval by the Institutional Review Board (KUH 1200104) of KUMC, and the need for informed consent from the patients was waived.

Basic patient characteristics are summarized in Table 1. Patients' medical records were reviewed at enrollment to obtain demographic, clinical, and laboratory data, and the diagnosis of LC $(\mathrm{N}=46)$ was made by hepatologists based on a combination of clinical and radiological results, such as splenomegaly, esophageal varix, and liver surface nodularity. Aspartate aminotransferase/alanine aminotransferase (AST/ALT), AST-to-platelet ratio index (APRI), and FIB-4 were calculated using laboratory data $[4,28]$. TE using FibroScan was performed by a well-trained technician, and TE results with $>10$ valid measurements and a success rate of $>60 \%$ and interquartile range (IQR) $\leq 30 \%$ were considered reliable $[8,11]$. Simultaneously, residual serum samples were collected following routine blood tests and stored at $-70^{\circ} \mathrm{C}$ until use, as described previously [18]. 
Table 1. Basic patient characteristics

\begin{tabular}{|c|c|}
\hline Parameter & Total $(\mathrm{N}=152)$ \\
\hline Age (yr) & $50.2 \pm 10.9$ \\
\hline \multicolumn{2}{|l|}{ Sex } \\
\hline Male (N, \%) & $97(63.8)$ \\
\hline Female (N, \%) & $55(36.2)$ \\
\hline LC (N, \%) & $46(30.3)$ \\
\hline TE (kPa) & $4.7(3.9-6.5)$ \\
\hline F0/1 (no or minimal) ( $\leq 7.0 \mathrm{kPa})(\mathrm{N}, \%)$ & $117(77.0)$ \\
\hline F2 (moderate) ( $\geq 7.1 \mathrm{kPa})(\mathrm{N}, \%)$ & $19(12.5)$ \\
\hline F3 (severe) ( $\geq 10.0 \mathrm{kPa})(\mathrm{N}, \%)$ & $7(4.6)$ \\
\hline F4 (cirrhosis) ( $\geq 13.0 \mathrm{kPa}$ ) (N, \%) & $9(5.9)$ \\
\hline AST (U/L) & $28.0(23.0-34.0)$ \\
\hline ALT (U/L) & $26.0(19.0-41.8)$ \\
\hline $\operatorname{ALP}(U / L)$ & $63.5(52.0-75.0)$ \\
\hline $\mathrm{GGT}(\mathrm{U} / \mathrm{L})^{*}$ & $37.0(22.0-67.5)$ \\
\hline $\mathrm{PLT}\left(\times 10^{9} / \mathrm{L}\right)^{\dagger}$ & $191.0(165.0-234.0)$ \\
\hline PT (sec/\%/INR) $)^{\ddagger}$ & $\begin{array}{c}13.2(12.7-13.7) / 99.0(92.0-107.5) / \\
1.0(0.9-1.1)\end{array}$ \\
\hline $\mathrm{aPTT}(\mathrm{sec})^{\S}$ & $34.8(32.2-37.2)$ \\
\hline AST/ALT & $1.1(0.8-1.4)$ \\
\hline $\mathrm{APRI}^{\dagger}$ & $0.3(0.2-0.5)$ \\
\hline $\mathrm{FIB}-4^{\dagger}$ & $1.4(1.0-2.1)$ \\
\hline ELF & $8.9(8.2-9.6)$ \\
\hline None/mild (<7.7) (N, \%) & $2(1.3)$ \\
\hline Moderate (7.7-9.8) (N, \%) & $124(81.6)$ \\
\hline Severe $(\geq 9.8)(N, \%)$ & $26(17.1)$ \\
\hline M2BPGi (COI) & $0.5(0.3-0.7)$ \\
\hline$-(<1.0)(\mathrm{N}, \%)$ & $134(88.2)$ \\
\hline $1+(1.0-3.0)(\mathrm{N}, \%)$ & $13(8.6)$ \\
\hline $2+(\geq 3.0)(N, \%)$ & $5(3.3)$ \\
\hline HBV DNA (copies/mL)" & $120.0(0.0-436.8)$ \\
\hline
\end{tabular}

Data are presented as mean $\pm \mathrm{SD}$, median (interquartile range), or number (percentage). Data of APRI, FIB-4, ELF, and M2BPGi according to the fibrosis grade based on TE are summarized in Supplemental Data Table S1.

${ }^{*}$ GGT levels were obtained from 71 patients at enrollment. ${ }^{\dagger} \mathrm{PLT}$ levels and APRI and FIB-4 scores were obtained from 130 CHB patients at enrollment. †PT levels were obtained from $112 \mathrm{CHB}$ patients at enrollment. ${ }^{\S} \mathrm{aPTT}$ levels were obtained from $11 \mathrm{CHB}$ patients at enrollment. "HBV DNA levels were obtained from $137 \mathrm{CHB}$ patients at enrollment.

Abbreviations: LC, liver cirrhosis; TE, transient elastography; $\mathrm{kPa}$, kilopascal; AST, aspartate aminotransferase; ALT, alanine aminotransferase; ALP, alkaline phosphatase; GGT, gamma-glutamyltransferase; PLT, platelet count; PT, prothrombin time; INR, international normalized ratio; aPTT, activated partial thromboplastin time; APRI, AST-to-platelet ratio index; FIB-4, fibrosis-4; ELF, enhanced liver fibrosis; M2BPGi, Mac-2 binding protein glycosylation isomer; COI, cut-off index; HBV, hepatitis B virus; DNA, deoxyribonucleic acid; SD, standard deviation.

\section{ELF and M2BPGi measurement}

ELF was measured using the ADVIA Centaur XP automated immunoanalyzer (Siemens Diagnostics, Tarrytown, NY, USA). The ELF score was calculated using the following equation: 2.278+ $0.851 \ln (\mathrm{HA})+0.751 \ln (\mathrm{PIIINP})+0.394 \ln (\mathrm{TIMP}-1)$, and was interpreted as follows: none/mild ( $<7.7)$, moderate (7.7-9.8), severe ( $\geq 9.8$ ) [12]. M2BPGi was measured using the HISCL-5000 immunoanalyzer (Sysmex, Kobe, Japan), based on a two-step sandwich chemiluminescent enzyme immunoassay [29]. The M2BPGi value was calculated as follows: cut-off index $(\mathrm{COI})=$ (M2BPGi-negative control)/(positive control-negative control) $[14,18]$. The M2BPGi value was interpreted as follows: $\mathrm{COI}>1.0$ was considered positive $(1+, 1.0 \leq \mathrm{COl}<3.0 ; 2+, \mathrm{COI} \geq 3.0)$ [29].

\section{Non-invasive clinical algorithms}

We applied two non-invasive clinical algorithms: a TE-first algorithm (TE/ELF or TE/M2BPGi approach) and a biomarker-first algorithm (ELF/TE or M2BPGi/TE approach) [25]. Based on the $T E$ results, the degree of $L F$ was dichotomized as advanced $(F \geq 3)$ or mild $(F \leq 2)$. Using biomarkers, manufacturer-claimed cut-off values with a high negative likelihood ratio (NLR) were selected to predict advanced LF: ELF $\geq 9.8$ and M2BPGi $\geq 3.0$ (Table 2).

In the TE-first algorithm, the low-risk group was defined as $\mathrm{TE} \leq \mathrm{F} 2$, and in the biomarker-first algorithm, the low-risk group was defined as ELF $<9.8$ and $M 2 B P G i<3.0$. If patients were not classified into the low-risk group by each algorithm, they were divided into concordant and discordant groups depending on the combined TE and biomarker results. The concordant group was defined as high risk ( $T E \geq F 3$; $E L F \geq 9.8$ or $M 2 B P G i \geq 3.0$ ), and LB was simulated only in the discordant group. Recently suggested clinical algorithms do not consider LB if the first risk assessment indicates low risk [25, 27]. However, we simulated LB consideration in the TE or biomarker low-risk group when the TE and biomarker values were discordant, not to lose cases with advanced LF in this group.

\section{Statistical analysis}

Data are expressed as mean \pm standard deviation (SD), median (interquartile range), or number (percentage). A normal distribution was evaluated using the Shapiro-Wilk test. The performances of ELF and M2BPGi in predicting advanced LF were evaluated by receiver operating characteristic (ROC) curve analysis. The area under the curve (AUC) with 95\% confidence interval $(\mathrm{Cl})$, sensitivity $(95 \% \mathrm{Cl})$, specificity $(95 \% \mathrm{Cl})$, positive likelihood ratio, and NLR of manufacturer-claimed cut-off values for ELF and M2BPGi were estimated by ROC curve analysis to pre- 
Table 2. Manufacturer-claimed cut-off values for ELF and M2BPGi to predict mild LF ( $F \leq 2$ by $T E$ ) and advanced LF ( $F \geq 3$ by TE)

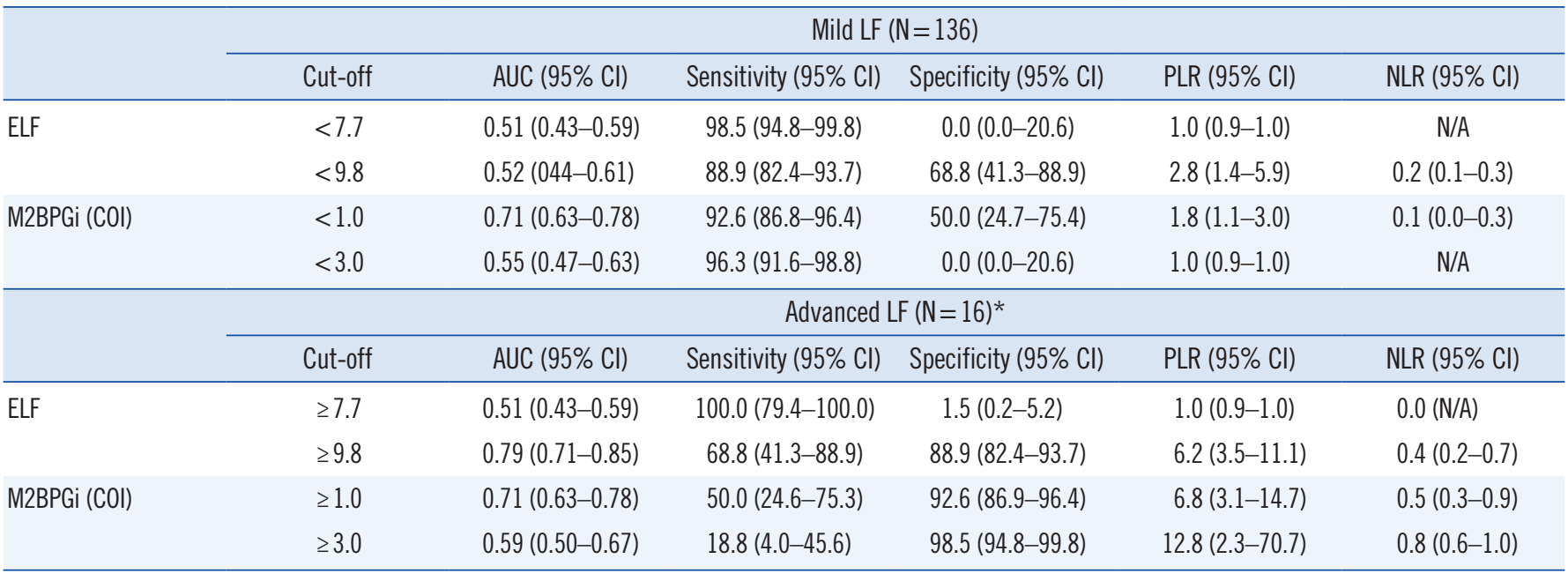

*See Supplemental Data Fig. 3.

Abbreviations: ELF, enhanced liver fibrosis; M2BPGi, Mac-2 binding protein glycosylation isomer; LF, liver fibrosis; TE, transient elastography; AUC, area under the curve; $\mathrm{Cl}$, confidence interval; PLR, positive likelihood ratio; NLR, negative likelihood ratio; N/A, not available; COI, cut-off index.

Table 3. Concordance among TE, ELF, and M2BPGi to predict mild LF ( $F \leq 2$ by TE) and advanced LF ( $F \geq 3$ by TE)

\begin{tabular}{lcccccc}
\hline & & Mild LF (N=136) & Advanced LF (N=16) & $P$ & Concordance (95\% Cl) & Kappa (95\% Cl) \\
\hline ELF & $<9.8$ & 121 & 5 & $<0.001^{*}$ & $86.8(72.7-102.9)$ & $0.45(0.25-0.65)$ \\
\multirow{2}{*}{ M2BPGi (COI) } & $\geq 9.8$ & 15 & 11 & & & \\
& $<3.0$ & 134 & 13 & $0.009^{\dagger}$ & $90.1(75.7-106.6)$ & $0.25(-0.00-0.50)$ \\
& $\geq 3.0$ & 2 & 3 & & & \\
\hline
\end{tabular}

${ }^{*}$ Chi-squared test. ${ }^{\dagger}$ Fisher's exact test.

Abbreviations: TE, transient elastography; ELF, enhanced liver fibrosis; M2BPGi, Mac-2 binding protein glycosylation isomer; LF, liver fibrosis; Cl, confidence interval; COI, cut-off index.

dict mild $L F(F \leq 2$ by $T E)$ and advanced $L F(F \geq 3$ by $T E)$. The Kruskal-Wallis test was used to compare values of APRI, FIB-4, ELF, and M2BPGi among fibrosis grades by TE (Supplemental Data Table 1). Chi-squared and Fisher's exact tests were used to compare the categorical variables; ELF and M2BPGi grades among fibrosis grades by TE, between advanced LF by TE and ELF, between advanced LF by TE and M2BPGi, the expected number of LBs between ELF and M2BPGi, and the expected number of LBs between the original and each modified approach. Concordance with $95 \% \mathrm{Cl}$ and Cohen's kappa with 95\% $\mathrm{Cl}$ between TE and ELF and between TE and M2BPGi were estimated. Cohen's kappa was interpreted as follows: none $(<0.01)$, slight $(0.01-0.2)$, fair $(0.21-0.4)$, moderate $(0.41-$ $0.6)$, substantial (0.61-0.8), and nearly perfect $(>0.81$ ) concordance [30]. We compared the added value of sensitivity across various combinations of TE, ELF, and M2BPGi using the concordance rate of TE, ELF, and M2BPGi as an analytical gold standard. In low-risk groups, the discordance rate between the
TE/ELF and TE/M2BPGi approaches and between the ELF/TE and M2BPGi/TE approaches was compared. We considered that a sequential approach with a low discordance rate in the low-risk group would be a suitable option for use in clinical practice. Statistical analysis was performed using MedCalc Statistical Software version 20 (MedCalc Software Ltd., Ostend, Belgium; https://www.medcalc.org; 2021). G*Power software version 3.1.9.7 (Franz Faul, University of Kiel, Kiel, Germany) was used to calculate the sample size based on statistical power. $P<0.05$ was considered statistically significant.

\section{RESULTS}

The concordance among TE, ELF, and M2BPGi to predict mild and advanced LF are presented in Table 3. The concordance rate between TE and ELF was $86.8 \%$ and that between TE and M2BPGi was $90.1 \%$, showing similar results for both biomarkers. The concordance between TE and ELF was moderate 

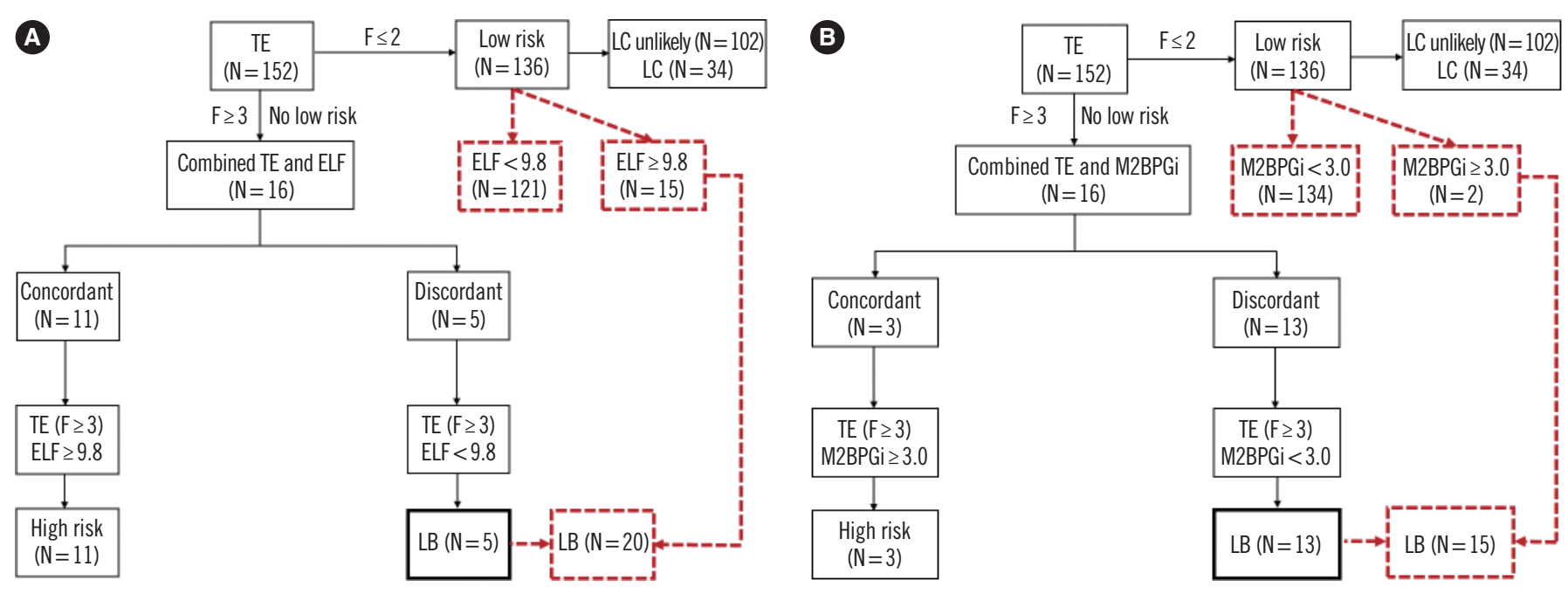

Fig. 1. Application of the TE-first algorithm. (A) TE/ELF approach. (B) TE/M2BPGi approach. The expected number of LBs was lower in the TE/M2BPGi approach than in the TE/ELF approach, with no statistical difference (9.9\% [15/152] vs. 13.2\% [20/152], $P=0.398)$. The discordance rate in the low-risk group was significantly lower in the TE/M2BPGi approach than in the TE/ELF approach (1.5\% [2/136] vs. $11.0 \%[15 / 136], P=0.002)$.

Abbreviations: TE, transient elastography; LC, liver cirrhosis; ELF, enhanced liver fibrosis; M2BPGi, Mac-2 binding protein glycosylation isomer; LB, liver biopsy.
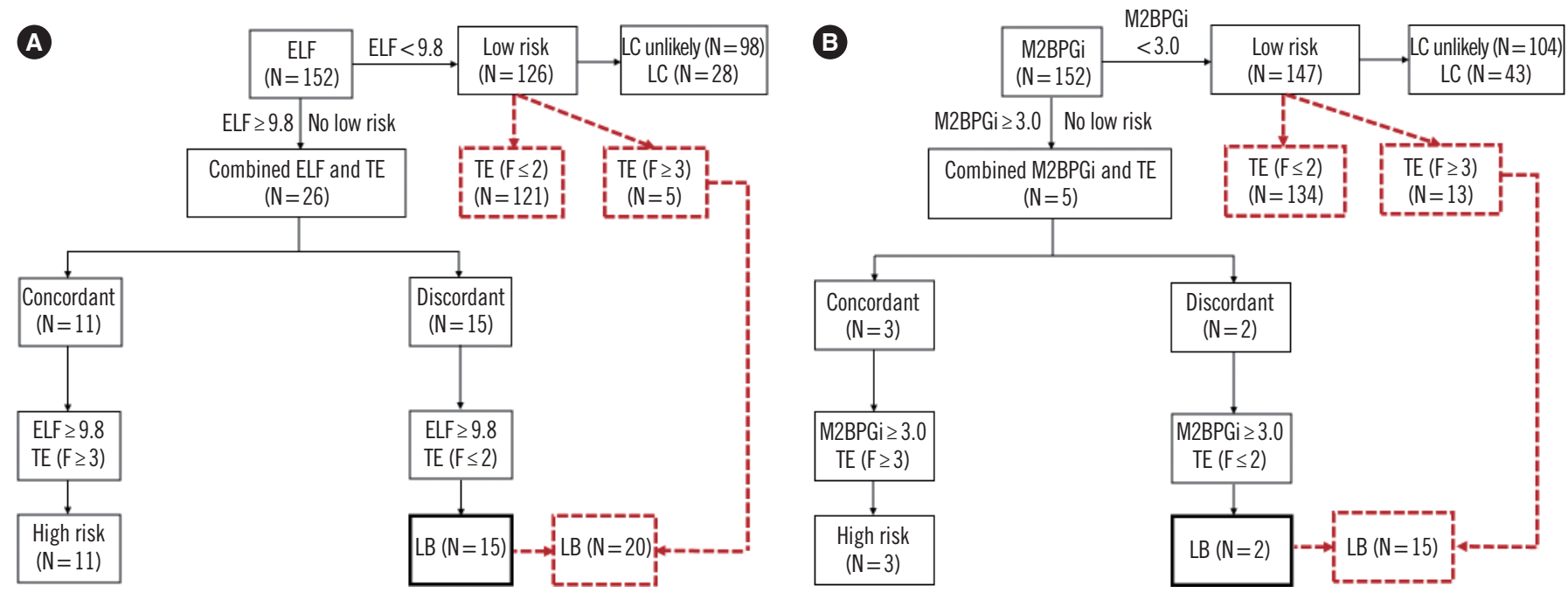

Fig. 2. Application of the serum biomarker-first algorithm. (A) ELF/TE approach. (B) M2BPGi/TE approach. The expected number of LBs was lower in the M2BPGi/TE approach than in the ELF/TE approach (9.9\% [15/152] vs. 13.2\% [20/152], $P=0.398$ ). The discordance rate in the low-risk group was lower in the ELF/TE approach than in the M2BPGi/TE approach, with no statistical difference (3.9\% [5/126] vs. $8.8 \%[13 / 147], P=0.118)$.

Abbreviations: see Fig. 1.

(kappa $=0.45$ ), whereas that between TE and M2BPGi was fair (kappa $=0.25)$.

In ROC curve analysis, the performances of ELF and M2BPGi in predicting advanced LF did not differ significantly; however, when the manufacturer-claimed cut-off values ( $\geq 9.8$ for ELF and $\geq 3.0$ for M2BPGi) were applied, their performances differed significantly ( $P=0.002)$ (Supplemental Data Fig. 2). The concordance rate of TE, ELF, and M2BPGi was 80.9\% ( $N=123)$.
When it was applied as an analytical gold standard (TE+ELF+ M2BPGi), three patients had advanced LF. In ROC curve analysis, the AUC was 0.97 for TE+ELF, 1.00 for TE+M2BPGi, and 0.99 for ELF+M2BPGi, showing a significant difference between $\mathrm{TE}+\mathrm{ELF}$ and TE+M2BPGi $(P=0.004)$.

The application of the TE-first algorithm, with the TE/ELF and TE/M2BPGi approaches, is shown in Fig. 1. Except for the TE low-risk group $(N=136)$, when using the sequential combina- 
tion of TE and biomarkers, the expected number of LBs was lower in the TE/ELF approach than in TE/M2BPGi approach, with no significant difference (3.3\% [5/152] vs. 8.6\% [13/152], $P=0.059$ ). In the TE low-risk group, the discordance rate between TE and biomarker results was significantly lower in the TE/M2BPGi approach than in the TE/ELF approach $(1.5 \%$ [2/136] vs. $11.0 \%$ [15/136], $P=0.002)$. The overall expected number of LBs was lower in the TE/M2BPGi approach than in the TE/ELF approach, with no significant difference $(9.9 \%$ [15/152] vs. 13.2\% [20/152], $P=0.398$ ).

The application of the serum biomarker-first algorithm, with the ELF/TE and M2BPGi/TE approaches, is shown in Fig. 2. Except for the low-risk groups based on each biomarker ( $N=126$ for ELF and $\mathrm{N}=147$ for M2BPGi), using the sequential combination of a biomarker and TE, the expected number of LBs was significantly lower in the M2BPGi/TE approach than in the ELF/ TE approach (1.3\% [2/152] vs. 9.9\% [15/152], $P=0.002)$. In the low-risk group based on each biomarker, the discordance rate between the biomarker and TE results was lower in the ELF/TE approach than in the M2BPGi/TE approach, with no significant difference (3.9\% [5/126] vs. 8.8\% [13/147], $P=0.118)$. The overall expected number of LBs was lower in the M2BPGi/ TE approach than in the ELF/TE approach (9.9\% [15/152] vs. $13.2 \%$ [20/152], $P=0.398$ ), with similar results for the two clinical algorithms.

\section{DISCUSSION}

This is the first study to apply non-invasive clinical algorithms using ELF and M2BPGi for the assessment of LF in CHB patients. Using sequential approaches combining TE and the two serum biomarkers, the final LB candidates ranged from $9.9 \%$ to $13.2 \%$ of the total of $152 \mathrm{CHB}$ patients, reserving LB only for patients with discordant results. In both the TE-first and serum biomarker-first algorithms, the expected number of LBs was the same, regardless of the test sequence applied. Between the two biomarkers, the expected number of LBs was lower when M2BPGi was applied than when ELF was applied, although the difference was not significant ( $P=0.398)$.

In a previous study, the optimal cut-off values for ELF to predict advanced $L F(F \geq 3$ by $L B, N=151)$ ranged from 8.4 (sensitivity of $95 \%$ ) to 10.8 (specificity of $92 \%$ ) [21]. With these ELF cut-off values, LB candidates among $\mathrm{CHB}$ patients ranged from $21.2 \%$ ( $N=18 / 85$, validation cohort) to $25.2 \%$ ( $N=60 / 238$, training cohort) [21]. In another recent study, a non-invasive clinical algorithm using ELF, FIB-4, or TE was proposed to pre- dict advanced LF in patients with NAFLDs [31]. Combined ELF ( $>10.83$ ) and TE (>11.45 $\mathrm{kPa}$ ) results increased the specificity to $97.9 \%$ and the positive predictive value to $91.7 \%$ when compared with ELF alone, and the sequential use of FIB-4 (>2.67) and ELF (>9.34) increased the sensitivity to 95.9\% [31]. To incorporate non-invasive tests into clinical practice, the simplest strategy is to begin with a test that has a high NLR to rule out high-risk cases [25]. We applied the manufacturer-claimed cutoff values to predict advanced LF (ELF $\geq 9.8$ and M2BPGi $\geq 3.0$ ), with NLRs of 0.4 and 0.8 , respectively. Although cut-off values with higher NLRs were obtained in this study population, studyspecific cut-off values cannot be generalized to other clinical settings. Accordingly, manufacturer-claimed cut-off values are more relevant in clinical practice and allow easy and objective interpretation, especially when used by non-specialists.

Using the manufacturer-claimed cut-off values, the concordance rates between TE and ELF and between TE and M2BPGi were similar, but their agreement was different. Kappa values can be influenced by several factors, such as prevalence and bias, and are reduced in case of a high prevalence index and high bias index [30]. The prevalence and bias indices of M2BPGi were greater than those of ELF, resulting in a low kappa value and explaining the difference in agreement (ELF vs. M2BPGi, 0.72 vs. 0.86 for the prevalence index, 0.06 vs. 0.07 for the bias index).

The European Association for Study of Liver-Asociacion Latinoamericana para el Estudio del Higado (EASL-ALEH) clinical practice guidelines state that non-invasive tests should be conducted prior to therapy by non-specialists to ensure that patients with severe fibrosis/cirrhosis are referred to a disease-specific specialist and that non-invasive approaches, such as serum biomarker or TE, can be used as first-line tests to assess the risk of severe fibrosis/cirrhosis [24]. The expected numbers of LBs were lower in both algorithms using M2BPGi (TE/M2BPGi and M2BPGi/TE approaches) than in those using ELF (TE/ ELF and ELF/TE approaches). Notably, the discordance rate in the low-risk group was the lowest when the TE/M2BPGi approach was used (1.5\% [2/136]). ELF and M2BPGi showed different diagnostic performance. The sensitivity of M2BPGi was significantly higher than that of ELF for predicting mild LF, whereas it was lower than that of ELF for predicting advanced LF; thus, ELF and M2BPGi seem to be suitable for screening and confirmatory test, respectively. However, the high NLR of M2BPGi was related to the low number of false positives in the low-risk group. A low discordance rate is important in clinical practice to minimize the risk of losing or neglecting cases of ad- 
vanced LF in the first-line test. The AUC of TE+M2BPGi was significantly higher than that of TE+ELF to predict advanced LF based on TE+ELF+M2BPGi (Supplemental Data Fig. 2). Taken together, among the four sequential approaches, the TE/M2BPGi approach seems to be the most relevant option in clinical practice. Our finding is in line with the recent NAFLD clinical practice guidelines from Korea, which suggest the TE-first approach as one of the sequential approaches in a non-invasive clinical algorithm [27].

Although TE is a first-line test for non-invasive assessment of LF, TE results should be interpreted with caution along with other test results. High AST or ALT levels may affect M2BPGi or ELF results as well as TE results [9, 14, 24, 31]. Although high AST or ALT levels may be a cause of discordance in each algorithm, very high AST or ALT levels were not observed in our study population. The non-invasive clinical algorithm using combined TE and ALT results has been suggested for treatment-naïve hepatitis $B$ virus (HBV)-infected patients, with a cut-off value of TE for LB of 6-9 kPa in case of a normal ALT level and of 6-12 kPa in case of an elevated ALT level [24]. TE should be used to detect LF only in HBV-infected patients with HBV DNA >2,000 IU/mL and a normal ALT level; in patients with very high ALT levels ( $>10 \times$ the upper normal limit), it is necessary to combine serum biomarkers and TE for predicting LF [24]. Boursier, et al. [32] proposed a stepwise algorithm using easy LF test (eLIFT), including age, sex, gamma-glutamyl transferase, AST, platelets, and prothrombin time, and then FibroMeter (FM) with vibrationcontrolled TE (VCTE). VCTE was indicated in patients with an eLIFT score $>8$. The sensitivity of the eLIFT-FM ${ }^{\text {VCTE }}$ algorithm (first-line eLIFT, second-line FM ${ }^{\mathrm{VCTE}}$ ) was $76.1 \%$ for advanced LF and 92.1\% for cirrhosis [32].

In this study, LC patients ranged from $22.2 \%$ to $29.3 \%$ in the low-risk groups: $25.0 \%$ (34/136) in the TE low-risk group, and $22.2 \%(28 / 126)$ and $29.3 \%(43 / 147)$ in the ELF and M2BPGi low-risk groups, respectively. They all had been diagnosed before study enrollment and received antiviral treatment. The presence of LC may not exactly reflect the current pathological condition of the liver. $L F$ is a dynamic, bidirectional process that has an inherent capacity for recovery and remodeling [1, 3, 33, 34]. As $L F$ is reversible by treatment, regression from $L C$ to early $L F$ or resolution of early LF to a nearly healthy liver is possible [1, 3]. Accordingly, except for treatment-naïve patients, LC patients can be included in TE low-risk and/or serum biomarker low-risk groups, as shown in this study. Our data also indicated that both TE-first and serum biomarker-first approaches can be used not only for the initial diagnosis or assessment of LC and LF, but also for the assessment of LF, if successfully treated, during its dynamic process. In each approach, follow-up and risk profile reassessment would be needed in the low-risk group regardless of the ELF or M2BPGi results. The total sample size was 152 in this study. To compare the algorithms, chi-squared and Fisher exact tests were mainly used. In the statistical power analysis, the estimated sample size ranged from 122 to 191 when assuming effect size of 0.3 , $\alpha$ error probability of 0.05 , power ( $1-\beta$ error probability) from 0.8 to 0.95 , and df of 3 for the chi-squared test [35]. For the sample size of 152, the power was approximately 0.92, indicating a sufficient sample size for Fisher exact test [36].

This study has several limitations. First, the study was performed in a limited number of CHB patients; the clinical algorithms using ELF and M2BPGi should be explored in patients with other liver diseases. Second, LB was not performed, but only simulated in the study population [19]. As TE is widely used to assess LF in many institutions, including ours, it was difficult and impractical to enroll patients with LB results. Considering the current strategies for non-invasive risk stratification that reserve LB only for patients with indeterminate results, our data provide an insightful strategy using readily available serum biomarkers [25]. However, more advanced and appropriate noninvasive algorithms, combining clinical and laboratory data (including biomarkers), are awaited. Third, we focused on applying and comparing non-invasive clinical algorithms using ELF and M2BPGi; thus, other conventional serum biomarkers, such as AST/ALT, APRI, or FIB-4, as well as HBV DNA levels were not considered. Both ELF and M2BPGi are novel biomarkers specific for LF assessment, and their levels can be interpreted objectively with suggested cut-off values. Accordingly, compared with conventional biomarkers, these biomarkers would be more beneficial for and applicable to initial screening that can be performed even by non-specialists to identify patients at risk of LF. Fourth, the purpose of this study was not to explore the diagnostic performance or accuracy of each test method in parallel or in combination, but to implement each biomarker sequentially in existing non-invasive clinical algorithms. Fifth, a selection bias effect could not be excluded. In this retrospective study, patients with mild LF according to TE were more prevalent than those with advanced LF according to TE, and M2BPGi was more concordant with mild LF based on TE than ELF, showing more favorable results in the low-risk group. Although it may be required to compare the clinical utility of these biomarkers using the same numbers of patients with mild LF and advanced LF, such equally sized patient populations can neither be expected 
in nor reflect the real clinical setting.

In conclusion, this was the first study to compare two non-invasive clinical algorithms using sequential approaches combining TE and the serum biomarkers ELF and M2BPGi. In each algorithm, regardless of the test sequence, both ELF and M2BPGi comparably reduced the expected number of LBs. Both ELF and M2BPGi could be used in initial screening tests to assess LF in CHB patients, although they showed different diagnostic performance. If priority is given to the capability of not losing advanced LF cases in the low-risk group, the sequential analysis of TE and M2BPGi seems to be the most practical approach to assess LF. Implementing novel serum biomarkers into non-invasive clinical algorithms should be further evaluated in patients with various other liver diseases.

\section{ACKNOWLEDGEMENTS}

None declared.

\section{AUTHOR CONTRIBUTIONS}

Hur M designed the study, analyzed the data, and wrote the manuscript; Park $\mathrm{M}$ analyzed the data and wrote the manuscript; Moon HW, Choe WH, and Lee $\mathrm{CH}$ participated in subject enrollment and study protocol; all authors reviewed and approved the final manuscript.

\section{CONFLICTS OF INTEREST}

None declared.

\section{RESEARCH FUNDING}

None.

\section{ORCID}

Mina Hur

Mikyoung Park

Hee-Won Moon

Won Hyeok Choe

Chae Hoon Lee https://orcid.org/0000-0002-4429-9978 https://orcid.org/0000-0002-4815-6958 https://orcid.org/0000-0001-9509-6073 https://orcid.org/0000-0002-8019-5412 https://orcid.org/0000-0001-7722-9004

\section{REFERENCES}

1. Rockey DC, Bell PD, Hill JA. Fibrosis—a common pathway to organ in- jury and failure. N Engl J Med 2015;372:1138-49.

2. World Health Organization. At last a global response to viral hepatitis. https://www.who.int/bulletin/volumes/88/11/10-011110/en/ (Updated on Feb 2020).

3. Pellicoro A, Ramachandran P, Iredale JP, Fallowfield JA. Liver fibrosis and repair: immune regulation of wound healing in a solid organ. Nat Rev Immunol 2014;14:181-94.

4. Soresi M, Giannitrapani L, Cervello M, Licata A, Montalto G. Non invasive tools for the diagnosis of liver cirrhosis. World J Gastroenterol 2014; 20:18131-50.

5. Bravo AA, Sheth SG, Chopra S. Liver biopsy. N Engl J Med 2001;344: 495-500.

6. Rousselet MC, Michalak S, Dupré F, Croué A, Bedossa P, Saint-André JP, et al. Sources of variability in histological scoring of chronic viral hepatitis. Hepatology 2005;41:257-64.

7. Liu T, Wang X, Karsdal MA, Leeming DJ, Genovese F. Molecular serum markers of liver fibrosis. Biomark Insights 2012;7:105-17.

8. Kim SU, Kim BK, Han KH. Clinical application of liver stiffness measurement using transient elastography: a surgical perspective. Digestion 2013;88:258-65.

9. Tsochatzis EA, Gurusamy KS, Ntaoula S, Cholongitas E, Davidson BR, Burroughs AK. Elastography for the diagnosis of severity of fibrosis in chronic liver disease: a meta-analysis of diagnostic accuracy. J Hepatol 2011;54:650-9.

10. Kim MN, Kim SU, Kim BK, Park JY, Kim DY, Ahn SH, et al. Increased risk of hepatocellular carcinoma in chronic hepatitis B patients with transient elastography-defined subclinical cirrhosis. Hepatology 2015; 61:1851-9.

11. Friedrich-Rust M, Poynard T, Castera L. Critical comparison of elastography methods to assess chronic liver disease. Nat Rev Gastroenterol Hepatol 2016;13:402-11.

12. Lichtinghagen R, Pietsch D, Bantel H, Manns MP, Brand K, Bahr MJ. The Enhanced Liver Fibrosis (ELF) score: normal values, influence factors and proposed cut-off values. J Hepatol 2013;59:236-42.

13. Narimatsu H. Development of M2BPGi: a novel fibrosis serum glycobiomarker for chronic hepatitis/cirrhosis diagnostics. Expert Rev Proteomics 2015;12:683-93.

14. Shirabe K, Bekki Y, Gantumur D, Araki K, Ishii N, Kuno A, et al. Mac-2 binding protein glycan isomer (M2BPGi) is a new serum biomarker for assessing liver fibrosis: more than a biomarker of liver fibrosis. J Gastroenterol 2018;53:819-26.

15. Cho SY and Hur M. Mac-2 binding protein glycosylation isomer: emerging non-invasive serum marker for liver fibrosis. Ann Lab Med 2018;38: 289-90.

16. Tamaki N, Kurosaki M, Loomba R, Izumi N. Clinical utility of Mac-2 binding protein glycosylation isomer in chronic liver diseases. Ann Lab Med 2021;41:16-24.

17. Jang SY, Tak WY, Park SY, Kweon YO, Lee YR, Kim G, et al. Diagnostic efficacy of serum Mac-2 binding protein glycosylation isomer and other markers for liver fibrosis in non-alcoholic fatty liver diseases. Ann Lab Med 2021;41:302-9.

18. Moon HW, Park M, Hur M, Kim H, Choe WH, Yun YM. Usefulness of enhanced liver fibrosis, glycosylation isomer of Mac-2 binding protein, galectin-3, and soluble suppression of tumorigenicity 2 for assessing liver fibrosis in chronic liver diseases. Ann Lab Med 2018;38:331-7.

19. Jekarl DW, Choi H, Lee S, Kwon JH, Lee SW, Yu H, et al. Diagnosis of liver fibrosis with Wisteria floribunda agglutinin-positive Mac-2 binding protein (WFA-M2BP) among chronic hepatitis B patients. Ann Lab Med 2018;38:348-54.

20. Castéra L, Sebastiani G, Le Bail B, de Lédinghen V, Couzigou P, Alberti A. 
Prospective comparison of two algorithms combining non-invasive methods for staging liver fibrosis in chronic hepatitis C. J Hepatol 2010;52: 191-8.

21. Wong GL, Chan HL, Choi PC, Chan AW, Yu Z, Lai JW, et al. Non-invasive algorithm of enhanced liver fibrosis and liver stiffness measurement with transient elastography for advanced liver fibrosis in chronic hepatitis B. Aliment Pharmacol Ther 2014;39:197-208.

22. Petta S, Vanni E, Bugianesi E, Di Marco V, Cammà C, Cabibi D, et al. The combination of liver stiffness measurement and NAFLD fibrosis score improves the noninvasive diagnostic accuracy for severe liver fibrosis in patients with nonalcoholic fatty liver disease. Liver Int 2015;35: 1566-73.

23. Calès P, Boursier J, Lebigot J, de Ledinghen V, Aubé C, Hubert I, et al. Liver fibrosis diagnosis by blood test and elastography in chronic hepatitis C: agreement or combination? Aliment Pharmacol Ther 2017;45: 991-1003.

24. European Association for Study of Liver; Asociacion Latinoamericana para el Estudio del Higado. EASL-ALEH Clinical Practice Guidelines: non-invasive tests for evaluation of liver disease severity and prognosis. J Hepatol 2015;63:237-64.

25. Tapper EB and Lok ASF. Use of liver imaging and biopsy in clinical practice. N Engl J Med 2017;377:2296-7.

26. Yoneda M, Imajo K, Takahashi H, Ogawa Y, Eguchi Y, Sumida Y, et al. Clinical strategy of diagnosing and following patients with nonalcoholic fatty liver disease based on invasive and noninvasive methods. J Gastroenterol 2018;53:181-96.

27. Korean Association for the Study of the Liver. Nonalcoholic Fatty Liver Disease Clinical Practice Guidelines 2021. https://www.kasl.org/bbs/index.html?code=guide\&category=\&gubun=\&idx=\&page=1\&number $=46$ 30\&mode $=$ view\&order $=\&$ sort $=\&$ keyfield $=\& k e y=($ Updated on May 2021 $)$.
28. Sterling RK, Lissen E, Clumeck N, Sola R, Correa MC, Montaner J, et al. Development of a simple noninvasive index to predict significant fibrosis in patients with HIV/HCV coinfection. Hepatology 2006;43:1317-25.

29. Kuno A, Ikehara Y, Tanaka Y, Ito K, Matsuda A, Sekiya S, et al. A serum "sweet-doughnut" protein facilitates fibrosis evaluation and therapy assessment in patients with viral hepatitis. Sci Rep 2013;3:1065.

30. Sim J and Wright CC. The kappa statistic in reliability studies: use, interpretation, and sample size requirements. Phys Ther 2005;85:257-68.

31. Inadomi C, Takahashi H, Ogawa Y, Oeda S, Imajo K, Kubotsu Y, et al. Accuracy of the enhanced liver fibrosis test, and combination of the enhanced liver fibrosis and non-invasive tests for the diagnosis of advanced liver fibrosis in patients with non-alcoholic fatty liver disease. Hepatol Res 2020;50:682-92.

32. Boursier J, de Ledinghen V, Leroy V, Anty R, Francque S, Salmon D, et al. A stepwise algorithm using an at-a-glance first-line test for the noninvasive diagnosis of advanced liver fibrosis and cirrhosis. J Hepatol 2017;66:1158-65

33. Ellis EL and Mann DA. Clinical evidence for the regression of liver fibrosis. J Hepatol 2012;56:1171-80.

34. Marcellin P, Gane E, Buti M, Afdhal N, Sievert W, Jacobson IM, et al. Regression of cirrhosis during treatment with tenofovir disoproxil fumarate for chronic hepatitis B: a 5 year open-label follow up study. Lancet 2013;381:468-75.

35. Faul F, Erdfelder E, Lang AG, Buchner A. G*Power 3: a flexible statistical power analysis program for the social, behavioral, and biomedical sciences. Behav Res Methods 2007;39:175-91.

36. Kim HY. Statistical notes for clinical researchers: sample size calculation 2. Comparison of two independent proportions. Restor Dent Endod 2016;41:154-6. 